\title{
Enhancement of Sleep Quality and Stability Using Acoustic Stimulation During Slow Wave Sleep
}

\author{
Aminollah Golrou ${ }^{1}$, Ali Sheikhani ${ }^{1 *}$, Ali Motie Nasrabadi², Mohammad Reza Saebipour ${ }^{3}$ \\ 'Department of Biomedical Engineering, Science and Research Branch Islamic Azad University, Tehran, Iran \\ ${ }^{2}$ Department of Biomedical Engineering, Shahed University, Tehran, Iran \\ ${ }^{3}$ Department of Neuroscience, Birjand University of Medical Sciences, Birjand, Iran
}

\begin{abstract}
Background: One of the challenges today is that the quality of sleep has weakened by many external factors, which we are not even aware of and which directly affect sleep. Sleep quality has an essential role in maintaining the cognitive function and memory consolidation of individuals. So far, various studies have been done to improve the quality of sleep by using external electrical stimulation, vestibular and olfactory system stimulation.

Methods: In this study, the increase in sleep quality was considered by simultaneous acoustic stimulation in a deep sleep to increase the density of slow oscillations. Slow oscillations are the important events recorded in electroencephalography (EEG) and hallmark deep sleep. Acoustic stimulation of pink noise with random frequency ranging from 0.8 to $1.1 \mathrm{~Hz}$ was used to improve sleep quality.

Results: Eight healthy adults (mean age: $28.4 \pm 7.8$ years) studied in 3 nights under 3 conditions: accommodation night, stimulation night (STIM) and no stimulation night (SHAM), in counterbalanced order. Significant characteristics of the objective and subjective quality of sleep have been extracted from the acquired EEG and compared in the last 2 nights. Also, the arousal and cyclic alternating pattern characteristics have been measured to assess sleep stability over 2 nights of STIM and SHAM.

Conclusion: Our findings confirm this goal of the study that applying designed acoustic stimulation simultaneously in the slow wave sleep (SWS) stage increases the duration of deep sleep and ultimately improves overall sleep stability and quality.

Keywords: Sleep quality enhancement; Acoustic stimulation; Slow wave sleep; CAP \& arousals; Sleep stability; EEG.
\end{abstract}

*Correspondence to

Ali Sheikhani, Department of Biomedical Engineering,

Science and Research Branch Islamic Azad University, Tehran, Iran.

Email:

sheikhani_al_81@srbiau.ac.ir

Published online 20 December 2018

Citation: Golrou A, Sheikhani A, Motie Nasrabadi A, Saebipour MR. Enhancement of sleep quality and stability using acoustic stimulation during slow wave sleep. Int Clin Neurosci J. 2018;5(4):126-134. doi: 10.15171/icnj.2018.25.

\section{Introduction}

Sleep is the absence of consciousness, the relative susceptibility of sensory perception, and the inactivity of almost all voluntary muscles at rest, that cyclic process of different physiological modalities, as can be observed by electrophysiologicalmeasureslikeelectroencephalography (EEG). Sleep is essential for maintaining energy and restoration of the body. ${ }^{1}$ The measurements of sleep quality are important as sleep quality affects the quality of life ${ }^{2}$ and daytime function. ${ }^{3}$ There is no fixed definition for the term "sleep quality," and it is commonly used in sleep medicine. ${ }^{4}$ Sleep quality sometimes referred to as a combination of some parameters including total sleep time (TST), sleep onset latency (SOL), sleep efficiency, the degree of fragmentation, and some sleep instability markers such as cyclic alternating patterns (CAPs) and arousals. ${ }^{5}$

Although the quality of sleep is a complex structure and rigorous empirical assessment, the validity of current and future research efforts largely depend on the methods used to determine the parameters of the sleep quality. In most recent studies in the field of sleep quality, there are 2 subjective and objective views for quantifying the quality of sleep. ${ }^{4}$ From the subjective point of view, the sleep quality components and their importance are different for individuals. ${ }^{6}$ So, it is essential to measure subjective sleep quality by using a self-report based evaluation. Although different questionnaires have been used to determine subjective sleep quality, they have several limitations. For example, the widely used Pittsburgh Sleep Quality Index (PSQI) which is a measure of global sleep quality based on the retrospective assessment of respondents from a set of sleep measures, including TST, SOL, sleep disturbances, daytime dysfunction, sleep efficiency and use of sleeping medications. The client rates questions in each of these seven sleep sections and the answers are scored based on a 0 to 3 scale, whereby 3 represents the negative extreme on the Likert Scale. ${ }^{7}$ A "poor" sleeper indicated by the total

(C) 2018 The Author(s). This is an open access article distributed under the terms of the Creative Commons Attribution License (http:// creativecommons.org/licenses/by/4.0/), which permits unrestricted use, distribution, and reproduction in any medium, provided the original work is properly cited. 
sum of " 5 " or higher. The PSQI used in numerous studies in various types of older adult populations that supports high validity and reliability. ${ }^{8}$ The PSQI commonly used in studies as their only measure of sleep quality. ${ }^{8}$ Although there are several questions about the client's bedmate or roommate of the client, these are not scored. ${ }^{8}$

Sleep quality is also sometimes taken from some objective features acquired from polysomnography (PSG) which is the gold standard in sleep quality measurement. ${ }^{4}$ The description of sleep architecture from PSG recordings is usually done using the standardized Rechtschaffen and Kales (R\&K) scoring guidelines ${ }^{9}$ or the newly published version of rules by the American Academy of Sleep Medicine (AASM).$^{10}$ Also, many architectural measures can extract from PSG that reflect the objective sleep quality, such as the percentage or temporal amounts of the N1 stage, N2 stage, N3 stage (or slow wave sleep [SWS]) and rapid eye movement (REM) sleep. ${ }^{11}$ Due to the use of no self-report analogs, objective measurements have made with some indicators of sleep quality. Significant changes in the PSG variables such as total awakening time increment, falling asleep delay, SWSs reduction and TST decrement usually associated with poor sleep quality. ${ }^{11}$

In addition to traditional polysomnography features which has some limitations, other characteristics could consider for sleep quality and stability measurements ${ }^{11,12}$ such as CAP rate and Arousal Index (AI). ${ }^{5}$ Instability of NREM sleep can be measured using CAPs which derived from PSG data. ${ }^{4}$ It quantifies the occurrence of particular periodic types of EEG patterns in NREM sleep and the degree to which shifts occur between these patterns over time, referred to as the CAP rate. ${ }^{13} \mathrm{CAP}$ rate is an analysis of sleep depth because it is a measure of data occurring during predefined sleep periods. ${ }^{4}$ Therefore, it may have the potential to measure sleep quality and stability. ${ }^{4}$ An EEG arousal, which is suddenly frequency shift toward faster rhythms (theta, alpha, beta), shortly interrupts sleep continuity for at least 3 seconds ${ }^{5}$ and can be scored according to the American Sleep Disorder Association (ASDA) guidelines. ${ }^{14}$ The number of arousals in NREM sleep and the number of arousals per hour of sleep (AI) which are related to measuring of awakening considered as significant indicators of sleep quality and instability. ${ }^{5}$

The hallmark of slow-wave sleep (SWS) is slow oscillations (SOs) which are electrical oscillations with a spectral peak frequency of about $0.8 \mathrm{~Hz}$ and the most significant events (amplitude $>75 \mu \mathrm{V}$ ) recorded in normal EEG. ${ }^{15}$ Many studies have shown various functions of the SOs, the most important of which is their essential role in memory consolidation, ${ }^{16}$ homeostatic regulation of synaptic connectivity, ${ }^{17}$ hormonal changes that affect glucose regulation ${ }^{18}$ and synaptic potentials triggered by learning. ${ }^{19}$ One of the critical characteristics of measuring the objective quality of sleep is the number of SWS in a deep sleep. ${ }^{11}$ It has proven that the higher the number and frequency of the periods of SWS in a deep sleep, the higher the goals of objective sleep quality are realized. ${ }^{11}$

Brain stimulation during sleep has recently considered in many studies. ${ }^{20-27}$ The type of stimulation is applied, and the purpose of stimulation is different in these studies. Transcranial direct current stimulation (TDCS), ${ }^{23,24,27}$ transcranial magnetic stimulation (TMS), ${ }^{20,28}$ vestibular stimulation $^{25}$ and acoustic stimulation, ${ }^{15,29-36}$ have been extensively applied during sleep. Various approaches have been taken to apply these stimuli, including sleep slow waves induction, ${ }^{15,22,35,36}$ accelerated human sleep homeostasis, ${ }^{24}$ insomnia treatment, ${ }^{25}$ tinnitus treatment, ${ }^{31}$ memory consolidation,,$^{32,33,37}$ and sleep spindles enhancement. ${ }^{27,38}$ One of the most important advantages of acoustic stimulation to other types of stimuli is that it can implement easily and without disturbance to the subject, and there is also no concern for lateral side effects in the future (in contrast with TDCS and TMS). ${ }^{15,30,33}$

In this study, the idea of sleep quality and stability enhancement using acoustic stimulation in normal subjects investigated. The purpose of the present study was to determine whether acoustic stimulation applied in sleep can increase the duration of SWS and also reduces the occurrence of arousals and CAPs. To this end, we apply closed-loop acoustic stimulation simultaneously with the N3 stage of NREM sleep to increase the density of slow oscillations in a deep sleep. We examined changes in sleep quality and stability measures comparing one night of no stimulation (SHAM) to one night of acoustic stimulation (STIM) in counter-balanced order. Also, participants completed the PSQI questionnaire before and after sleep in both nights.

\section{Materials and Methods}

Subjects and Data Acquisition

Eight healthy adults (mean age: $28.4 \pm 7.8$ years) studied in 2 nights (with/with no stimulation) after one accommodation night. Full-night sleep EEG signals recorded from normal subjects in Birjand University of Medical Sciences (BUMS) sleep research laboratory. None of the participants had any medical disorder, neurological disorder, and were not taking any drug to sleep. EEG recorded from 12 channels placed according to the international 10-20 standard: Fp1, Fp2, Fz, T7, T8, C3, C4, Cz, Po7, Po8, Pz and Oz) referenced to the average of left and right mastoid electrodes. Electrode placement has done for each participant one hour before their usual sleep time. EEG recordings started at the subject's usual bedtime and terminated at their usual time of getting up in the morning. The sampling rate for the EEG recording was $250 \mathrm{~Hz}$, and EEG signals were filtered by $0.3 \mathrm{~Hz}$ high-pass filter and a low-pass filter of $35 \mathrm{~Hz}$. All 30-second epochs of 12 channels EEG signal scored by a neuroscientist expert according to the AASM. ${ }^{10}$

Three conditions for the entry of subjects volunteering to participate in this study were considered: First, subjects report normal sleep quality for the past month. To this 
end, all subjects completed the PSQI questionnaire. A total sum of " 5 " or smaller shows an average quality sleeper. ${ }^{8}$ Second, subjects experience at least three SWS stages with a duration of 15 minutes per 8 hours of nighttime. ${ }^{10}$ This point noted after a specialist does the data recorded on the second night and the scoring. Third, the subject's hearing system should be normal, because the auditory stimulation considered in this study. According to the standard hearing curves and auditory stimulation threshold applied in the present study, if the subject has an auditory test of 125 to $8000 \mathrm{~Hz}$ with a minimum hearing threshold (10) $\mathrm{dB}$, he will be recognized as a normal person. The above-mentioned auditory test conducted for all volunteer subjects in the research. Finally, according to the three conditions stated above, out of 12 volunteer subjects to participate in the study, eight eligible people were identified after the first night.

Data was recorded in 30-second epochs in 2 different nights at intervals of one week from eight subjects selected for this study. On the first night, only the matching of the individual with the recording environment considered and no data recorded. In the second night, the purpose was to collect the necessary information for the design of the system for SWS detection and to identify the normal sleep conditions of individuals (the second condition expressed in the previous paragraph). In the third night, application of acoustic stimulation simultaneously in deep sleep and its effect on SWS duration and sleep quality considered. The intended signal processing also took place in 2 steps. In the first step, using the data recorded on the second night, a system for SWS detection was designed, which should have a small delay in the data processing stage. The system was used for online SWS detection in the third night and applying simultaneous acoustic stimulation. The second stage of data processing, which coincides with the final recording on the third night, is used to determine the acoustic stimulation timing and uses the information obtained in the first stage of the process.

Data acquisition Hardware and Acoustic Stimulation The eWave 32D, with 32 channels of 24-bit recording with an input range of $2.5(\mathrm{v})$ and the sampling rate of 1000 $\mathrm{Hz}$ was used to record the EEG signal in this research. The electrodes of this device are inactive and the device connected to the computer via Wifi. Samsung's wireless curve speaker with PC connectivity also used for acoustic stimulation. This speaker was placed at a distance of 80 $\mathrm{cm}$ from the subject's head and above it. Also, a sound level meter (TES 1358 according to IEC60561) used for acoustic stimulation intensity calibration. Speaker sound volume was measured and calibrated before each stimulation night using this device (Figure 1).

For the design of a system that aims to increase the SWS duration by acoustic stimulation, stimulation parameters should investigate. By examining previous studies in this field ${ }^{20,34-44}$ the acoustic stimulation parameters that are

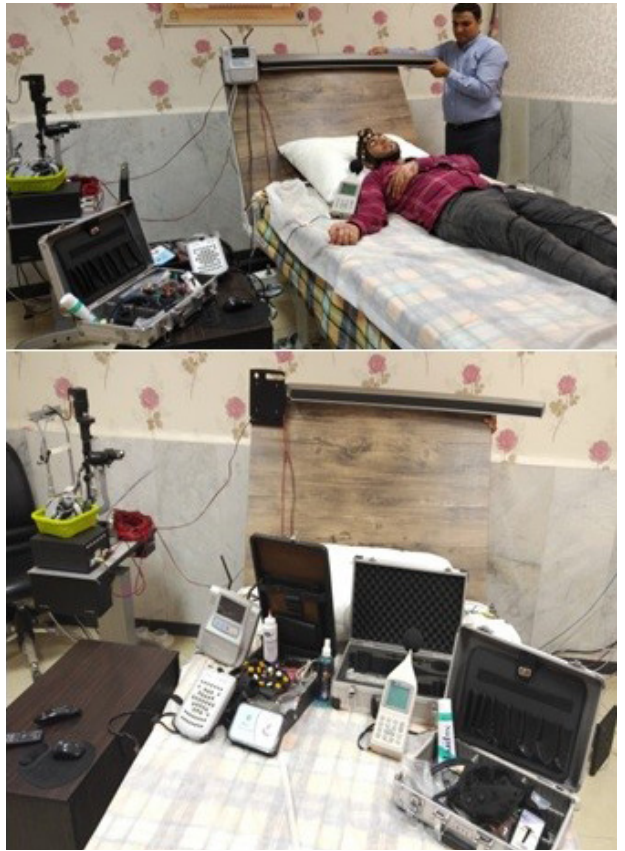

Figure 1. Data Acquisition Hardware Used in BUMS Sleep Research Lab.

effective in increasing the appearance of slow oscillations can determine. By optimizing effective factors, we can maximize it:

\section{Stimulation Intensity}

The acoustic stimulation intensity should be limited to prevent unwanted arousals according to a study by Terzano et $\mathrm{al}^{40}{ }^{40}$ acoustic perturbation with the intensity of 45 to $75 \mathrm{~dB}$ significantly increases the waking time after sleep onset, NREM and N2 stage durations, while decreasing the duration of SWS, REM and TST. As a result of this, acoustic stimulation in this intensity range not only does not help improve sleep quality but also reduces it.

\section{Stimulation Frequency}

The acoustic stimulation frequency should be determined randomly in order to avoid habituation on the auditory system $^{22}$ according to the study by Sokolov et $\mathrm{al}^{41}$ habits are described as a pattern of response reduction that occurs after sensory stimuli. So, when it comes to increasing slow oscillations, creating unique conditions for a degree of randomness to prevent the habit during stimulation is essential.

\section{Entrainment}

Any practice that aims and causes that frequencies of EEG fall into step with the frequency of a periodic stimulus corresponding to the intended brain-state is named brainwave entrainment. ${ }^{42}$ It has shown that acoustic stimulation produced entrainment of slow oscillations activity in neurons of anesthetized guinea pigs. ${ }^{43}$ According to the study by Ngo et a ${ }^{15}$ rhythmic acoustic 
stimulation applied to normal subjects can be used to entrain the brain's sleep EEG oscillatory events like slow oscillations.

According to the points mentioned above, acoustic stimulation of pink $1 / \mathrm{f}$ noise with random frequency ranging of its push from 0.8 to $1.1 \mathrm{~Hz}$ used in this study. Pink noise was used instead of white noise because it is softer and more comfortable for subjects to hear. Also, its frequency spectrum is similar to normal EEG. The random sound intensity applied between 20 and $60 \mathrm{~dB}$. Figure 2 depicts an example of the acoustic stimulation which applied in this study.

\section{Online Slow Wave Sleep Detection}

We have developed an automated fast real-time algorithm that can detect the SWS. Depending on the need for a high-speed system for SWS detection, feature extraction, and classification method has been used. In this system, due to previous studies ${ }^{44}$ and relatively good performance of frequency characteristics, this feature is used for SWS detection. As a result, the power of the EEG signal in 12 channels in the following subchannels considered as a feature: 0.5 to $2 \mathrm{~Hz}, 2$ to $4 \mathrm{~Hz}, 4$ to $8 \mathrm{~Hz}, 8$ to $12.5 \mathrm{~Hz}$, 12.5 to $15 \mathrm{~Hz}, 15$ to $18 \mathrm{~Hz}$ and 18 to $30 \mathrm{~Hz}$. A multi-layer perceptron (MLP) neural network with 12 neurons in the hidden layer has been used to classify the signals. These features extracted from all signals on the first night of data recording (second night). Fifty percent of the total epochs used for training, $10 \%$ used for training evaluation, and $40 \%$ used for classifier test. The SWS detection system then used for subjects in the second-night data acquisition (third night), which showed an average sensitivity of $81.4 \%$ in training and $78.8 \%$ in the classifier test. This system, based on tests carried out on sleep laboratory computer, is capable of detecting SWS within $300 \mathrm{~ms}$.

\section{Results}

\section{Subjective Sleep Quality Measure}

The mean PSQI global score was $5.8 \pm 2.8$ for subjects in the second night (without stimulation). The amount of this measure decreased on the third night (with stimulation) to $3.4 \pm 1.9$, which confirms the improvement in the quality of sleep expressed by subjects in the night of stimulation. The PSQI total scores were also significantly different between 2 nights, with higher scores for the STIM night. None of the subjects were aware of the effect of acoustic stimulation on the third night. Also, the first

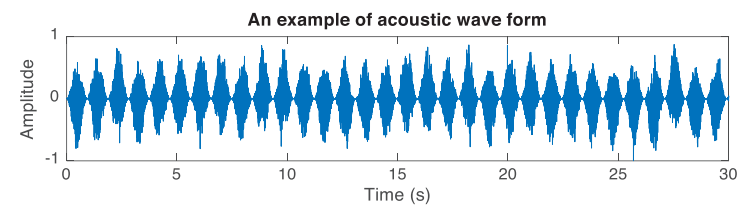

Figure 2. Pink Noise With Random Frequency Ranging From 0.8 to $1.1 \mathrm{~Hz}$ Applied in This Study. night there was an acoustic stimulation (to determine the maximum sound level). Also, there was at least one week gap between SHAM and STIM night, so reducing the amount of PSQI is not related to the subject's induction or adaptation. The overall PSQI reliability was high for the total score and for the all sub-items except for the "sleep quality" and "sleep disturbance," which were in the midrange.

\section{Objective Sleep Quality Measure}

Regarding objective sleep quality, one of the important parameters is the study of the number and duration of SWS. ${ }^{11}$ The number and duration of the SWS, the more the goals of good sleep are realized, in other words, the quality of sleep increases from the objective point of view. These goals include the homeostatic regulation of synaptic strength, ${ }^{22}$ memory consolidation, ${ }^{37,45}$ body movement control increase and the level of hormones in the body have previously reviewed. In some previous studies $^{29,30,32,36,37}$ acoustic stimulation has been applied to entrain slow oscillations, which are locked with a phase of slow waves, but in some other studies ${ }^{15}$ acoustic stimulation has been applied regardless of the phase of slow oscillations. Acoustic stimulation application, regardless of the slow oscillation phase, increases the duration of the SWS, while it does not affect the slow wave activity (SWA). Figure 3 depicts the SWA power histogram for a subject in the SHAM night and the STIM night. As shown in this figure, stimulation in the STIM did not have much effect on the average SWA power compared to the SHAM night. About the other subjects, the effect of stimulation is different, and so it can be argued that given the fact that the stimulation applied regardless of the up or downward
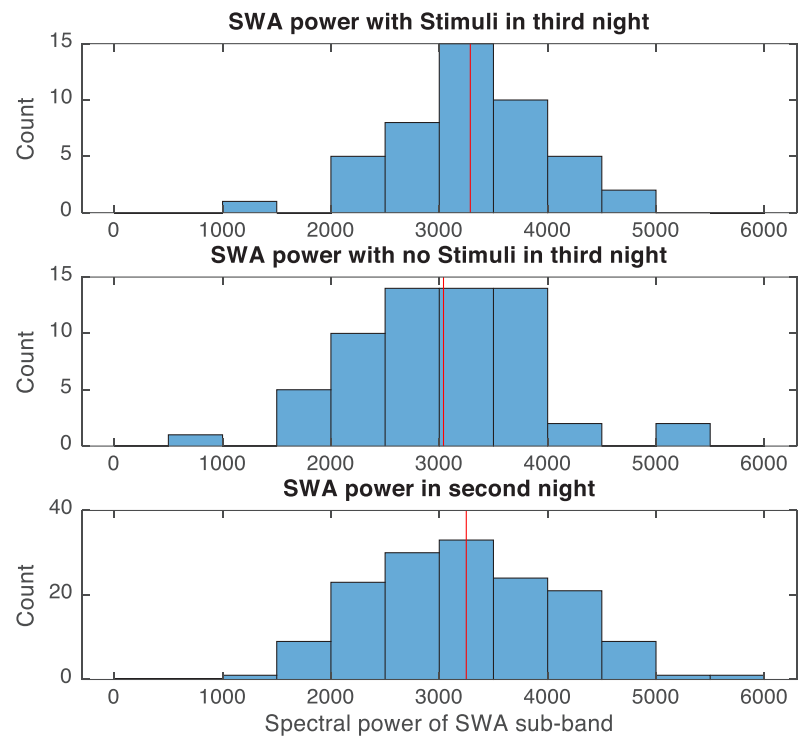

Figure 3. SWA Power Histogram for the Third Subject in Second Night (No Stimulation) and Third Night (With and Without Stimulation). 
phase of slow oscillation, we cannot comment on its effectiveness.

However, regarding the effect of stimulation on deep sleep, its effect on the SWS duration, as well as the maximum length of continuous SWS, is measured. Figure 4 shows the hypnogram chart of the third subject's sleep on the STIM night compared to the SHAM.

As seen, continuous SWS duration has increased in the STIM night compared to the SHAM, which can view from the point of objective sleep quality improvement. Also, in the STIM night, sleep continuity is more than SHAM, which means that the stage change density and successive returns to the previous stage are higher in the SHAM night compared to the STIM. From the viewpoint of sleep stability, the awakening rate was lower during STIM compared to the SHAM and applying stimulation increased sleep stability. In Tables 1 and 2, the results for the total SWS time and maximum SWS duration for the SHAM and STIM nights have reported.

By comparing the values presented in Table 1, it can conclude that the total SWS duration for each of the eight subjects participating in this study has increased by
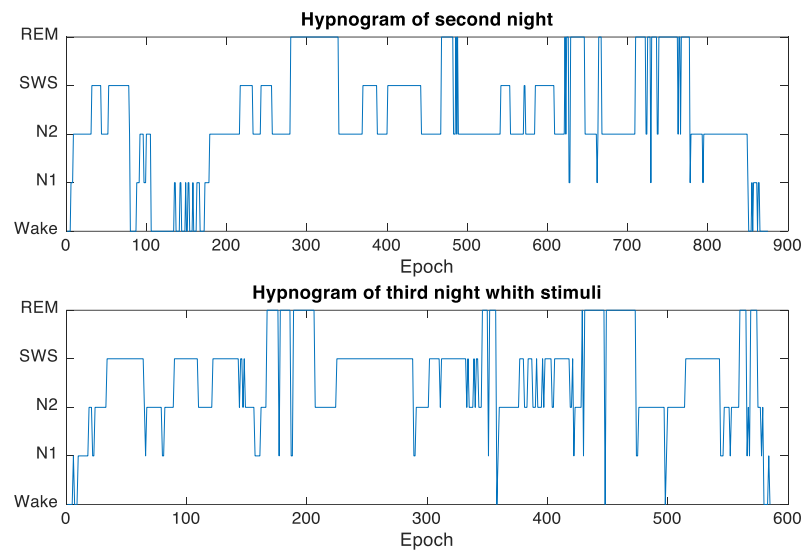

Figure 4. The Hypnogram Chart of The Third Subject's Sleep on the STIM Night Compared to the SHAM Night.

Table 1. Total SWS Duration for All Subjects in STIM \& SHAM Nights Compared to the TST

\begin{tabular}{lcccc}
\hline Subjects & $\begin{array}{c}\text { Total Sleep } \\
\text { Time (SHAM) } \\
(\mathbf{m i n})\end{array}$ & $\begin{array}{c}\text { Total SWS } \\
\text { Duration } \\
(\text { SHAM) }(\mathbf{m i n})\end{array}$ & $\begin{array}{c}\text { Total Sleep } \\
\text { Time (STIM) } \\
(\mathbf{m i n})\end{array}$ & $\begin{array}{c}\text { Total SWS } \\
\text { Duration } \\
(\text { STIM) }(\mathbf{m i n})\end{array}$ \\
\hline 1 & 448 & 131 & 400 & 160.5 \\
\hline 2 & 444.5 & 91 & 332 & 106.5 \\
\hline 3 & 363 & 118.5 & 437.5 & 192 \\
\hline 4 & 493 & 134.5 & 427 & 170.5 \\
\hline 5 & 423.5 & 119 & 326 & 144 \\
\hline 6 & 360.5 & 66.5 & 421.5 & 119.5 \\
7 & 479.5 & 143.5 & 428.5 & 182 \\
\hline 8 & 409 & 153.5 & 349 & 153 \\
\hline Average & 427.6 & 119.7 & 390.2 & 153.5 \\
\hline
\end{tabular}

applying stimulation. The ratio of SWS in the STIM night increased by $12 \%$ compared to the SHAM night.

In this research, three different characteristics have been measured to examine the effect of acoustic stimulation on the objective sleep quality and stability:

\section{Sleep Structural Characteristics}

These characteristics are related to the sleep hypnogram. A specialist extracts the duration of each sleep stage relative to the total sleep time (TST). In this regard, 6 characteristics of (N1 time/TST), (N2 time/TST), (N3 time/TST), (REM time/TST), (NREM time/TST), (N3 time/NREM time) are considered.

\section{Cyclic Alternating Pattern-Related Characteristics}

CAP as a marker of unstable sleep reflects a condition of consolidated sleep. Two characteristics of CAP time in total sleep time and CAP rate (ratio of CAP time to NREM sleep time) in this study considered. The characteristic values measured from data classified by a specialist. The higher the amount of CAP rate is related to the poorer sleep quality.

\section{Arousals Related Characteristics}

An arousal event often used as a measure for quantifying the sleep fragmentation that is related to both sleep quality and stability. Arousal scoring performed according to the American Sleep Disorder Academy (ASDA) guidelines. ${ }^{46}$ The following arousal related variables were measured: number of arousals in total sleep time (total arousals), and the number of arousals per hour of sleep (AI).

As a result, the average value of the mentioned above ten characteristics for eight subjects in 2 nights (STIM vs. SHAM) calculated. Figure 5 illustrates the obtained values graphically.

\section{Discussion}

In the present research, the primary goal is to detect the deep sleep stage online and simultaneously acoustically stimulate the SWS. By adjusting the acoustic stimulation parameters, the best possible result is the increase in the

Table 2. Maximum SWS Duration for All Subjects in STIM Night Compared to the SHAM Night

\begin{tabular}{lcc}
\hline \multirow{2}{*}{ Subjects } & \multicolumn{2}{c}{ Maximum SWS Duration $(\mathbf{m i n})$} \\
\cline { 2 - 3 } & SHAM & STIM \\
\hline 1 & 37.5 & 42.5 \\
2 & 21.5 & 41 \\
3 & 19.5 & 30.5 \\
4 & 39 & 40.5 \\
5 & 20.5 & 38 \\
6 & 12.5 & 31.5 \\
7 & 35 & 39.5 \\
8 & 41.5 & 46 \\
Average & 28.4 & 38.7 \\
\hline
\end{tabular}




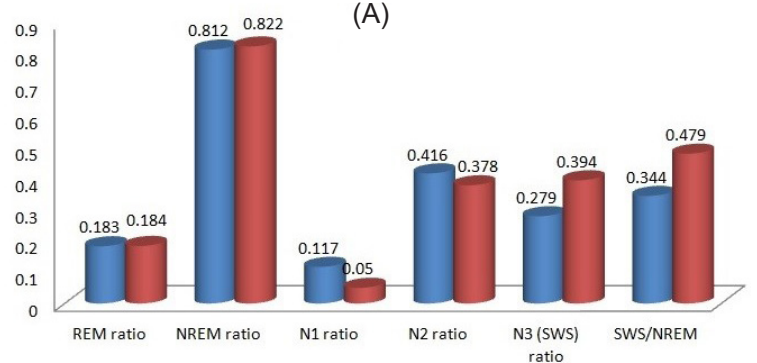

(B)

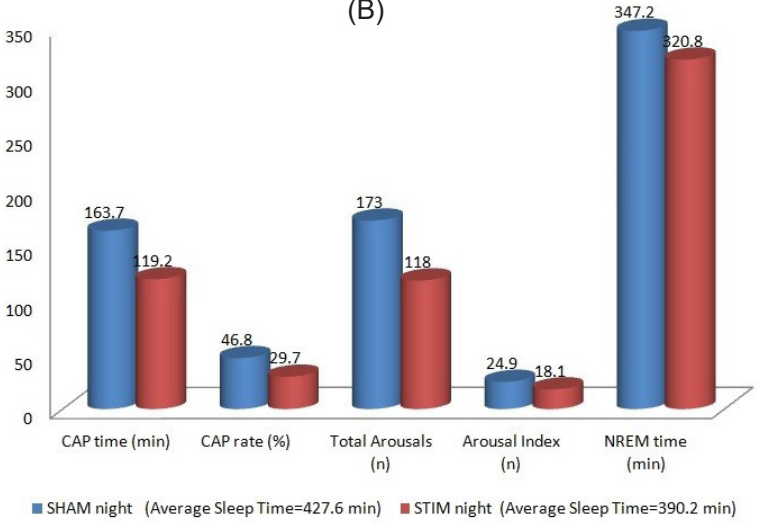

Figure 5. The Comparative Graph of the Average Obtained Values for 10 Variables Related to the Quality and Stability of the Subjects' Sleep Measured by the Expert on the SHAM and STIM Nights. (A): sleep structural variables. (B): CAP \& arousal related variables.

appearance of slow waves, as well as the reduction of CAPs and Arousals level in NREM sleep. In some previous studies, acoustic stimulation was applied before going to sleep ${ }^{29}$ or applied at a specified time without regard to the sleep stages. ${ }^{33,35,36}$ The use of acoustic stimulation in this way, firstly, causes an increase in one of the main poor sleep quality, the "SOL," which results in lower sleep quality, leading to reduced sleep efficiency (ratio of sleep time to time in bed). Also, acoustic stimulation in this way causes arousals, especially in the REM sleep and N1 and N2 stages of NREM sleep, which ultimately causes the subject to awaken, and the primary goal of this study is to go away. Experimental results confirm this goal of the study that applying acoustic stimulation simultaneously with SWS increases the duration of the emergence of slow waves and ultimately improves overall sleep stability and quality. Here we discuss our findings in three subsections:

\section{The Main Architecture of Sleep Remained Unchanged During and After Acoustic Stimulation}

The important criteria for the objective quantification of sleep quality are that the normal hypnogram structure should be observed to achieve normal sleep. ${ }^{11}$ In the normal hypnogram structure, the observance of the sequence of entering into different stages of sleep, as well as the duration to which each stage, is important. As seen in Figure 5A, the REM \& NREM ratios in 2 data acquisition's nights (STIM vs. SHAM) were not significantly altered.
In other words, the acoustic stimulation does not affect the overall sleep structure. The duration of N3 (or SWS) sleep stage, increased by approximately $12 \%$ applying stimulation, while the duration of REM sleep remained almost constant, and the $12 \%$ increase in N3 stage resulted in a reduction in total N1 \& N2 stages. In other words, the total duration of NREM sleep, as well as the REM duration, did not change over the entire duration of sleep. In this regard, it can argue that using designed stimulation in this study, sleep architecture remained unchanged and it is crucial concerning the sleep quality that stimulation does not disrupt the main structure of sleep.

\section{Increase in Overall and NREM Sleep Stability}

As indicated in the results section, the level of sleep stages in conjunction with STIM night is higher than SHAM. In other words, it can state that the number of successive returns to the previous stage in the STIM night has been reduced compared with the SHAM. Also, the amount of awakening during sleep in the STIM night is less than SHAM, and the duration of continuous deep sleep has increased significantly (see Figure 4 and Table 2). Also, due to the comparison of the $\mathrm{AI}$ and total arousals in the STIM night with SHAM night (Figure 5B), it is clear that the number of total arousals in the stimulation night decreased. In effect, arousal counting can be considered as a reliable indicator of sleep instability and has used for studying on related sleep disorders. ${ }^{12}$ Many factors including sensory stimulation can affect the appearance of arousals in sleep. Arousal variables related to awakening marked in this study and because implemented acoustic stimulation with an appropriate intensity measured at the first accommodation night, has not only increased the number of arousals but has reduced them. Also, the total awakening item in the PSQI Questionnaire, subjects in this study have stated that in the STIM night they woke up less and had a more stable sleep than SHAM night. Also, as known in the hypnograms obtained from STIM night and the comparison with SHAM night (Figure 5), the frequency of awakening is less than STIM night. The above results regarding the increase in overall and NREM sleep stability can compare with the recent study by Jobst et $\mathrm{al}^{47}$ that suggests a mechanism for the functional changes observed during SWS leading to increased sleep stability. Finally, we can declare that using acoustic stimulation applied in this study, the total stability of sleep has increased.

One of the other characteristics of sleep stability measured in this research is the CAP that are known as the measure of NREM instability. ${ }^{48,49}$ CAP stands as a marker of sleep instability, whereas the absence of CAP reflects a condition of consolidated sleep. An increase in CAPs has reported in normal subjects by increasing the level of acoustic stimulation using high sound intensity ${ }^{40}$ as well as those with insomnia. ${ }^{48}$ Also, the relationship between the rate of CAPs and the level of sleep arousals (which 
causes sleep awakening and instability) is also studied. ${ }^{50}$

As shown in Figure 5B, both the CAP time and CAP rate characteristics reduced significantly over STIM night. Of course, in the case of CAP time characteristic, there is some discussion in that the overall sleep time (TST) of the STIM night was lower than that of the SHAM night. However, it is important that the NREM sleep time was only reduced by about $10 \%$ at STIM night compared to the SHAM, while the CAP time characteristic decreased by about $50 \%$ in the STIM night compared to the SHAM.

\section{Sleep Quality Enhancement Due to the Increase in SWS Duration}

One of the most prominent effects of acoustic stimulation in this study is the increase in SWS duration (See Figure $5 \mathrm{~A})$. SWS ratio as one of the measured characteristics for objective sleep quality, increased at STIM night by about $12 \%$ compared to the SHAM, and this increase in the ratio of SWS associated with a decrease in the ratio of $\mathrm{N} 1$ and N2 stages. It is important that increasing the duration of SWS in the NREM stage can affect the quality of sleep. Regarding the increase in SWS duration, we focused on induced slow oscillations in SWS. As investigated before, ${ }^{35}$ if acoustic stimulation applied phase-locked to slow oscillations, it significantly increased and amplified endogenous slow oscillations, and subsequently, the SWA increased. This type of stimulation has been applied in some of the previous studies for such purposes as memory consolidation, ${ }^{37}$ concomitant memory improvement, ${ }^{32}$ vocabulary memory enhancement, ${ }^{30,36}$ sleep strengthening and signs of its immune-supportive function. ${ }^{29}$ However, inducing slow oscillations using the phase locked excitation also bear the risk of the emergence of epilepsy, suggesting the presence of healthy brain mechanisms that counter developing hyper synchronicity during slow waves. ${ }^{35}$ However, in this study, designed acoustic stimulation has been applied non-phase locked to slow oscillations. SWA canceled by this type of stimulation or diminished due to the phase cancellation which reduces the likelihood of seizure and epilepsy.

Furthermore, the topography of induced slow oscillations is very similar to the topographic profile of natural slow oscillations, which is characterized by a frontal predominance. By examining the results of the fp1 channel, it has determined that the induced slow oscillations in this study are also more likely to appear in the frontal channels than other channels. This conclusion confirms the results obtained by Tononi et $\mathrm{a}^{22}$ that the increase in slow waves shows topography consistent with the frontal predominance of normal sleep.

From the perspective of sleep quality, induced slow waves prolong the duration of deep sleep and thus can increase the quality of sleep. Acoustic enhancement of slow waves and memory consolidation has already been considered in several studies, ${ }^{30,32,33,36,37}$ but as far as we know, improving sleep quality using acoustic stimulation has not yet been studied. Increase in SWS duration and the decrease in frequency of awakening (decrease in total arousals) are 2 important hypnogram features that confirm sleep quality enhancement using applied acoustic stimulation in our study.

Also, according to studies on the comparison of objective sleep quality measurement metrics using Polysomnography, the rate of CAPs has been introduced as an appropriate indicator of objective sleep quality measurement. ${ }^{5}$ Both clinical and experimental studies ${ }^{51}$ have established a significant correlation between the subjective estimates of sleep quality and the ratio of CAP time to the non-REM Sleep time. Therefore, reducing the CAP time and CAP rate (See Figure 5B) by acoustic stimulation in this study and affecting sleep stability has also been effective in improving sleep quality.

\section{Conclusion}

In conclusion, closed-loop acoustic stimulation applied simultaneously with deep sleep improved both subjective and objective sleep quality measures. By using acoustic stimulation, the total PSQI score has decreased that related to the subjective sleep quality enhancement and total arousals and CAP rates has also decreased that related to the objective sleep quality enhancement. We showed that using designed acoustic stimulation simultaneously with the deep sleep, increases the duration of SWS and ultimately improves overall sleep stability and quality while the main architecture of sleep remained unchanged.

\section{Conflict of Interest Disclosures}

The authors declare that they have no conflict of interests.

\section{Ethical Statement}

Not applicable.

\section{References}

1. 1.Cirelli C, Tononi G. Is sleep essential? PLoS Biol. 2008;6(8):e216. doi: 10.1371/journal.pbio.0060216.

2. Iliescu EA, Coo H, McMurray MH, Meers CL, Quinn MM, Singer MA, et al. Quality of sleep and health-related quality of life in haemodialysis patients. Nephrol Dial Transplant. 2003;18(1):126-32.

3. Alapin I, Fichten CS, Libman E, Creti L, Bailes S, Wright J. How is good and poor sleep in older adults and college students related to daytime sleepiness, fatigue, and ability to concentrate? J Psychosom Res. 2000;49(5):381-90.

4. Krystal AD, Edinger JD. Measuring sleep quality. Sleep Med. 2008;9 Suppl 1:S10-7. doi: 10.1016/s1389-9457(08)70011-x.

5. Terzano MG, Parrino L, Spaggiari MC, Palomba V, Rossi $M$, Smerieri A. CAP variables and arousals as sleep electroencephalogram markers for primary insomnia. Clin Neurophysiol. 2003;114(9):1715-23.

6. Harvey AG1, Stinson K, Whitaker KL, Moskovitz D, Virk H. The subjective meaning of sleep quality: a comparison of individuals with and without insomnia. Sleep. 2008;31(3):38393.

7. Yi H, Shin K, Shin C. Development of the sleep quality scale. J Sleep Res. 2006;15(3):309-16. doi: 10.1111/j.13652869.2006.00544.x. 
8. Smyth C. The Pittsburgh Sleep Quality Index (PSQI). https:// consultgeri.org/try-this/general-assessment/issue-6.1.pdf. Published 2012.

9. Himanen SL, Hasan J. Limitations of Rechtschaffen and Kales. Sleep Med Rev. 2000;4(2):149-67. doi: 10.1053/ smrv.1999.0086.

10. Novelli L, Ferri R, Bruni O. Sleep classification according to AASM and Rechtschaffen and Kales: effects on sleep scoring parameters of children and adolescents. J Sleep Res. 2010;19(1 Pt 2):238-47. doi: 10.1111/j.1365-2869.2009.00785.x.

11. Rosipal R, Lewandowski A, Dorffner G. In search of objective components for sleep quality indexing in normal sleep. Biol Psychol. 2013;94(1):210-20. doi: 10.1016/j. biopsycho.2013.05.014.

12. Golrou A, Maghooli K, Amiri AM, Mankodiya K, Ghaemi $\mathrm{K}$, editors. Automatic sleep apnea detection using fuzzy logic. 2015 IEEE Signal Processing in Medicine and Biology Symposium (SPMB); 2015 12-12 Dec. 2015.

13. Halasz P, Bodizs R. Dynamic Structure of NREM Sleep. London: Springer; 2013. doi: 10.1007/978-1-4471-4333-8.

14. Diagnostic classification of sleep and arousal disorders. 1979 first edition. Association of Sleep Disorders Centers and the Association for the Psychophysiological Study of Sleep. Sleep. 1979;2(1):1-154.

15. Ngo HV, Claussen JC, Born J, Molle M. Induction of slow oscillations by rhythmic acoustic stimulation. J Sleep Res. 2013;22(1):22-31. doi: 10.1111/j.1365-2869.2012.01039.x.

16. Diekelmann S, Wilhelm I, Born J. The whats and whens of sleep-dependent memory consolidation. Sleep Med Rev. 2009;13(5):309-21. doi: 10.1016/j.smrv.2008.08.002.

17. Bellesi M, Riedner BA, Garcia-Molina GN, Cirelli C, Tononi G. Enhancement of sleep slow waves: underlying mechanisms and practical consequences. Front Syst Neurosci. 2014;8:208. doi: 10.3389/fnsys.2014.00208.

18. Tasali E, Leproult R, Ehrmann DA, Van Cauter E. Slowwave sleep and the risk of type 2 diabetes in humans. Proc Natl Acad Sci U S A. 2008;105(3):1044-9. doi: 10.1073/ pnas.0706446105

19. Huber R, Ghilardi MF, Massimini M, Ferrarelli F, Riedner BA, Peterson MJ, et al. Arm immobilization causes cortical plastic changes and locally decreases sleep slow wave activity. Nat Neurosci. 2006;9(9):1169-76. doi: 10.1038/nn1758.

20. Marshall L, Born J. Brain stimulation during sleep. Sleep Med Clin. 2011;6(1):85-95. doi: 10.1016/j.jsmc.2010.12.003.

21. Coenen A. Subconscious Stimulus Recognition and Processing During Sleep. Psyche. 2010;16(2):90-7.

22. Tononi G, Riedner BA, Hulse BK, Ferrarelli F, Sarasso S. Enhancing sleep slow waves with natural stimuli. Medicamundi. 2010;54(2):73-9.

23. Lustenberger C, Boyle MR, Alagapan S, Mellin JM, Vaughn BV, Frohlich F. Feedback-Controlled Transcranial Alternating Current Stimulation Reveals a Functional Role of Sleep Spindles in Motor Memory Consolidation. Curr Biol. 2016;26(16):2127-36. doi: 10.1016/j.cub.2016.06.044.

24. Reato D, Gasca F, Datta A, Bikson M, Marshall L, Parra LC. Transcranial electrical stimulation accelerates human sleep homeostasis. PLoS Comput Biol. 2013;9(2):e1002898. doi: 10.1371/journal.pcbi.1002898.

25. Krystal AD, Zammit GK, Wyatt JK, Quan SF, Edinger JD, White DP, et al. The effect of vestibular stimulation in a four-hour sleep phase advance model of transient insomnia. J Clin Sleep Med. 2010;6(4):315-21.

26. Javadi Arjomand AH. Memory modulation by offline consolidation and transcranial direct current stimulation. University College London; 2011.

27. Loo C, Martin D. Transcranial direct current stimulation.
In: Hamani C, Holtzheimer P, Lozano AM, Mayberg $\mathrm{H}$, eds. Neuromodulation in Psychiatry. Wiley; 2016. doi:10.1002/9781118801086.ch12.

28. Massimini $M$, Ferrarelli F, Esser SK, Riedner BA, Huber $R$, Murphy $M$, et al. Triggering sleep slow waves by transcranial magnetic stimulation. Proc Natl Acad Sci U S A. 2007;104(20):8496-501. doi: 10.1073/pnas.0702495104.

29. Besedovsky L, Ngo HV, Dimitrov S, Gassenmaier C, Lehmann $\mathrm{R}$, Born J. Auditory closed-loop stimulation of EEG slow oscillations strengthens sleep and signs of its immunesupportive function. Nat Commun. 2017;8(1):1984. doi: 10.1038/s41467-017-02170-3.

30. Ngo HV, Martinetz T, Born J, Molle M. Auditory closedloop stimulation of the sleep slow oscillation enhances memory. Neuron. 2013;78(3):545-53. doi: 10.1016/j. neuron.2013.03.006.

31. Pedemonte $M$, Testa $M$, Diaz $M$, Suarez-Bagnasco D. The Impact of Sound on Electroencephalographic Waves during Sleep in Patients Suffering from Tinnitus. Sleep Sci. 2014;7(3):143-51. doi: 10.1016/j.slsci.2014.09.011.

32. Papalambros NA, Santostasi G, Malkani RG, Braun R, Weintraub S, Paller KA, et al. Acoustic Enhancement of Sleep Slow Oscillations and Concomitant Memory Improvement in Older Adults. Front Hum Neurosci. 2017;11:109. doi: 10.3389/fnhum.2017.00109.

33. Ong JL, Lo JC, Chee NI, Santostasi G, Paller KA, Zee PC, et al. Effects of phase-locked acoustic stimulation during a nap on EEG spectra and declarative memory consolidation. Sleep Med. 2016;20:88-97. doi: 10.1016/j.sleep.2015.10.016.

34. Czisch M, Wetter TC, Kaufmann C, Pollmacher T, Holsboer F, Auer DP. Altered processing of acoustic stimuli during sleep: reduced auditory activation and visual deactivation detected by a combined fMRI/EEG study. Neuroimage. 2002;16(1):2518. doi: 10.1006/nimg.2002.1071.

35. Ngo HV, Miedema A, Faude I, Martinetz T, Molle M, Born J. Driving sleep slow oscillations by auditory closedloop stimulation-a self-limiting process. J Neurosci. 2015;35(17):6630-8. doi: 10.1523/jneurosci.3133-14.2015.

36. van Poppel EAM. Predicting brainwaves: The influence of auditory Closed-loop cueing during Slow Oscillation Upstates on Vocabulary Memory [thesis]. Amsterdam: University of Amsterdam; 2016.

37. Leminen MM, Virkkala J, Saure E, Paajanen T, Zee PC, Santostasi G, et al. Enhanced Memory Consolidation Via Automatic Sound Stimulation During Non-REM Sleep. Sleep. 2017;40(3). doi: 10.1093/sleep/zsx003.

38. Schabus M, Dang-Vu TT, Heib DP, Boly M, Desseilles M, Vandewalle G, et al. The Fate of Incoming Stimuli during NREM Sleep is Determined by Spindles and the Phase of the Slow Oscillation. Front Neurol. 2012;3:40. doi: 10.3389/ fneur.2012.00040.

39. Wilson C, Agafonov RV, Hoemberger M, Kutter S, Zorba A, Halpin J, et al. Kinase dynamics. Using ancient protein kinases to unravel a modern cancer drug's mechanism. Science. 2015;347(6224):882-6. doi: 10.1126/science.aaa1823.

40. Terzano MG, Parrino L, Fioriti G, Orofiamma B, Depoortere H. Modifications of sleep structure induced by increasing levels of acoustic perturbation in normal subjects. Electroencephalogr Clin Neurophysiol. 1990;76(1):29-38.

41. Hay JC, Sokolov YN. Perception and the conditioned reflex. Am J Psychol. 1966;79(1):166.

42. Brainwave entrainment. Wikipedia website. https:// en.wikipedia.org/wiki/Brainwave_entrainment.

43. Gao L, Meng X, Ye C, Zhang H, Liu C, Dan Y, et al. Entrainment of slow oscillations of auditory thalamic neurons by repetitive sound stimuli. J Neurosci. 2009;29(18):6013-21. 
doi: 10.1523/jneurosci.5733-08.2009.

44. Su BL, Luo Y, Hong CY, Nagurka ML, Yen CW. Detecting slow wave sleep using a single EEG signal channel. J Neurosci Methods. 2015;243:47-52. doi: 10.1016/j. jneumeth.2015.01.023.

45. Diekelmann S, Biggel S, Rasch B, Born J. Offline consolidation of memory varies with time in slow wave sleep and can be accelerated by cuing memory reactivations. Neurobiol Learn Mem. 2012;98(2):103-11. doi: 10.1016/j.nlm.2012.07.002.

46. Mathur R, Douglas NJ. Frequency of EEG arousals from nocturnal sleep in normal subjects. Sleep. 1995;18(5):330-3.

47. Jobst BM, Hindriks R, Laufs H, Tagliazucchi E, Hahn G, PonceAlvarez A, et al. Increased Stability and Breakdown of Brain Effective Connectivity During Slow-Wave Sleep: Mechanistic Insights from Whole-Brain Computational Modelling. Sci Rep. 2017;7(1):4634. doi: 10.1038/s41598-017-04522-x.
48. Chouvarda I, Mendez MO, Rosso V, Bianchi AM, Parrino L, Grassi A, et al. CAP sleep in insomnia: new methodological aspects for sleep microstructure analysis. Conf Proc IEEE Eng Med Biol Soc. 2011;2011:1495-8. doi: 10.1109/ iembs.2011.6090341.

49. Rizzi M, Sarzi-Puttini P, Atzeni F, Capsoni F, Andreoli A, Pecis $M$, et al. Cyclic alternating pattern: a new marker of sleep alteration in patients with fibromyalgia? J Rheumatol. 2004;31(6):1193-9.

50. Terzano MG, Mancia D, Salati MR, Costani G, Decembrino A, Parrino L. The cyclic alternating pattern as a physiologic component of normal NREM sleep. Sleep. 1985;8(2):137-45.

51. Golrou A, Sheikhani A, Nasrabadi AM, Saebipour MR. Detecting slow wave sleep and rapid eye movement stage using cortical effective connectivity. Turk J Elec Eng Comp Sci. 2018;26(6):2779-92. 